\title{
Genome-Wide Sequencing as a First-Tier Screening Test for Short Tandem Repeat Expansions
}

Indhu-Shree Rajan-Babu ${ }^{1}{ }^{*}$, Junran Peng ${ }^{1}$, Readman Chiu ${ }^{2}$, IMAGINE Study ${ }^{1}$, CAUSES Study ${ }^{1}$, Arezoo Mohajeri ${ }^{1}$, Egor Dolzhenko ${ }^{3}$, Michael A. Eberle ${ }^{3}$, Inanc Birol ${ }^{1,2}$, Jan M. Friedman ${ }^{1}$

${ }^{1}$ Department of Medical Genetics, University of British Columbia, and Children's \& Women's Hospital, Vancouver, BC, Canada

${ }^{2}$ Michael Smith Genome Sciences Centre, British Columbia Cancer Agency, Vancouver, BC, Canada

${ }^{3}$ Illumina Inc, San Diego, CA, US

*Address correspondence to Indhu Shree Rajan Babu, Ph.D., Department of Medical Genetics, University of British Columbia, British Columbia, Vancouver, Canada. Tel: +1-604-875-2000 ext. 5980, Email: indhu.babu@bcchr.ca 


\section{ABSTRACT}

2 Short tandem repeat (STR) expansions cause several neurological and neuromuscular disorders.

3 Screening for STR expansions in genome-wide (exome and genome) sequencing data can enable

4 diagnosis, optimal clinical management/treatment, and accurate genetic counselling of patients

5 with repeat expansion disorders. We assessed the performance of lobSTR, HipSTR, RepeatSeq,

6 ExpansionHunter, TREDPARSE, GangSTR, STRetch, and exSTRa - bioinformatics tools that

7 have been developed to detect and/or genotype STR expansions - on experimental and simulated

8 genome sequence data with known STR expansions aligned using two different aligners, Isaac

9 and BWA. We then adjusted the parameter settings to optimize the sensitivity and specificity of

the STR tools and fed the optimized results into a machine-learning decision tree classifier to

11 determine the best combination of tools to detect full mutation expansions with high diagnostic

12 sensitivity and specificity. The decision tree model supported using ExpansionHunter's full

13 mutation calls with those of either STRetch or exSTRa for detection of full mutations with

14 precision, recall, and F1-score of $90 \%, 100 \%$, and $95 \%$, respectively.

We used this pipeline to screen the BWA-aligned exome or genome sequence data of 306

16 families of children with suspected genetic disorders for pathogenic expansions of known disease

17 STR loci. We identified 27 samples, 17 with an apparent full-mutation expansion of the $A R$,

18 ATXN1, ATXN2, ATXN8, DMPK, FXN, HTT, or TBP locus, nine with an intermediate or

19 premutation allele in the FMRI locus, and one with a borderline allele in the ATXN2 locus. We

20 report the concordance between our bioinformatics findings and the clinical PCR results in a

21 subset of these samples. Implementation of our bioinformatics workflow can improve the

22 detection of disease STR expansions in exome and genome sequence diagnostics and enhance

23 clinical outcomes for patients with repeat expansion disorders. 


\section{INTRODUCTION}

25 Expansions of short tandem repeats (STRs; tandemly repeated arrays of 1-6 base pair (bp)

26 sequence motifs $^{1}$ ) can cause several neurological and neuromuscular disorders ${ }^{2}$. Accurate

27 genotyping (i.e., the determination of the number of copies of repeat units in an STR) is critical

28 to the molecular diagnosis of STR expansion disorders as repeat length usually shows a positive

29 correlation with severity and negative correlation with age of onset of clinical symptoms ${ }^{3}$.

30 Repeat length also determines an STR's allelic class (normal, NL; intermediate, IM;

31 premutation, PM; or full-mutation, FM), which may differ with respect to associated disease

32 phenotype ${ }^{3 ; 4}$. For example, the FMRl (MIM 309550) PM (55-200 CGG repeats) increases the

33 risk for primary ovarian insufficiency (MIM 311360) and tremor/ataxia syndrome (MIM

34 300623). In contrast, FMR1 FM (>200 CGG repeats) causes fragile X syndrome (MIM 300624),

35 the most frequent Mendelian cause of intellectual disability ${ }^{5}$. PM and IM (also known as

36 "mutable NL") alleles that are meiotically unstable can expand into pathogenic FM in a single

37 generation, while NL alleles rarely, if ever, do so ${ }^{6 ;}$. Expanded alleles tend to further increase in

38 repeat length during intergenerational transmission, and, as a result, genetic anticipation (the

39 earlier and more severe manifestation of disease symptoms with each successive generation) is

40 common in repeat expansion disorders ${ }^{8}$.

$41 \quad$ Clinical laboratories typically use polymerase chain reaction (PCR) or Southern blot (SB)

42 (alone or in combination) to characterize expansions at known disease STR loci ${ }^{9}$. Although

43 highly sensitive in detecting and genotyping STR expansions, PCR and SB tests have several

44 limitations. They are time- and labor-intensive, require extensive optimization, and do not permit

45 concurrent analyses of more than a handful of STR loci. Next-generation sequencing (NGS), on

46 the other hand, enables exome- and genome-wide characterization of STRs. Several algorithms 
47 have recently been developed to analyse STRs in NGS data ${ }^{1 ; 10-14}$. The incorporation of

48 bioinformatics tools to screen for STR expansions may permit the diagnosis of repeat expansion

49 disorders during routine diagnostic exome or genome sequencing, allow accurate genetic

50 counseling of affected individuals and their families, and improve clinical outcomes.

The currently-available STR analysis algorithms have different attributes that determine

52 their utility and sensitivity in detecting and characterizing repeat expansions in NGS data (Table

53 1). Methods like STRetch ${ }^{11}$ and exSTRa ${ }^{12}$ identify STR expansions via case-control analysis,

54 with a caveat of either underestimating the repeat lengths of some expanded STRs ${ }^{11}$ or not

55 genotyping STRs ${ }^{12}$. Methods that genotype STRs are known to perform better across certain

56 repeat length ranges depending on the read type evidence considered. For instance, tools relying

57 on reads that fully encompass an STR ("spanning reads") to compute repeat length ${ }^{15-17}$ can size

58 alleles within the length of an Illumina read (125-150 base pairs [bp]) but they perform poorly in

59 detecting pathogenic FM expansions that exceed read length. More recent methods ${ }^{1 ; 10 ; 18 ; 19}$ that

60 leverage on additional read types such as flanking or partially flanking reads (those that map to

61 unique flanking sequences), in-repeat reads (IRR; those that are entirely composed of STRs with

62 a mate that maps to the STR's flanking sequence), and/or IRR pairs (both reads of a pair

63 mapping to the STR) can size STRs that exceed read length. ExpansionHunter ${ }^{10 ; 19}$ and

64 GangSTR $^{18}$, in particular, enable the recovery of IRR and IRR pairs, which originate from an

65 expanded STR but may incorrectly map to other STR (or "off-target") regions with longer tracts

66 of the same repeat motif. By allowing the inclusion of off-target sites (OTS) in analysis,

67 ExpansionHunter and GangSTR facilitate sizing STRs that are longer than an Illumina

68 sequencing library fragment length (350-500 bp). 
In terms of utility, some of these methods can analyse STRs in both exome sequencing

70 (ES) and genome sequencing (GS) data ${ }^{11 ; 12 ; 18}$, while others are designed specifically for GS ${ }^{1 ; 10 ;}$

$71{ }^{19}$. Some tools have specific NGS data requirements; for example, ExpansionHunter is designed

72 for PCR-free GS, and exSTRa has only been extensively tested on bowtie- $2^{20}$ alignments. Also,

73 most methods have been recognized to perform less optimally on GC-rich STR expansions ${ }^{10 ; 12 .}$

74 These varied attributes and performance characteristics have led to the acknowledgment that a

75 single bioinformatics tool is less likely to be able to identify pathogenic STR expansions of all

76 repeat lengths and sequence content/composition in NGS data ${ }^{12}$. Recently, Tankard et al

77 recommended a consensus calling approach using at least two out of four tools (TREDPARSE ${ }^{1}$,

78 ExpansionHunter, STRetch, and exSTRa) to characterize expansions of known disease STRs ${ }^{12}$.

79 However, it is not clear which of these (or other) STR methods alone or in combination yield

80 optimal sensitivity and specificity.

In this study, we employed a decision tree classifier to identify the optimal tool(s) for

82 classifying expanded FM and non-expanded alleles at known disease STR loci with high

83 accuracy, precision, recall, and F1-score. We performed our analysis on the STR calls from nine

84 different tools $1 ; 10-12 ; 15 ; 17-19 ; 21$ made on the GS data of patients with well-characterized STR

85 expansions in one of eight different loci $(A R, A T N 1, A T X N 1, A T X N 3, D M P K, F M R 1, F X N$, or

$86 H T T)^{10}$ and simulated GS data harboring expansions of the GC-rich FMR2 or C9orf72 STR loci.

87 These data were aligned using two different aligners, Isaac ${ }^{22}$, an ultra-fast aligner, and BWA-

$88 \mathrm{MEM}^{23}$, recommended by the GATK best practices guidelines ${ }^{24}$ and widely used in GS

89 studies $^{25}$, to see if the choice of the aligner influences the performance of the STR methods.

90 First, we tested the classifier on the results generated by the implementation of tools using

91 default parameter settings. We then tweaked several parameters, such as the inclusion/exclusion 
92 of OTS and using a different FM repeat length threshold to define expansions at selected loci and

93 implementation of exSTRa with a control cohort, to optimize the sensitivity and specificity of the

94 STR tools included in this study. Once we established the parameters that yielded the best

95 results, we input the data generated with these settings into the classifier and found a significant

96 improvement in our model's ability to detect FMs compared to our default parameter assessment.

97 We then applied our decision tree model of STR algorithms to screen for expansions in known

98 disease STR loci in the GS or ES data of 306 families (patient-parent trios (patient and both

99 biological parents) or quads (patient, sibling, and both biological parents)) with a proband who is

100 suspected to have a genetic disorder.

\section{METHODS AND APPROACHES}

102 GS Datasets with a Known Repeat Expansion

103 The GS datasets with a known repeat expansion analysed in this study include the BWA and

104 Isaac alignments of: 1) the European Genome-phenome archive (EGA) dataset ${ }^{10}$

105 (EGAD00001003562), which consisted of data from 118 PCR-free GS of Coriell samples, each

106 with an $A R, A T N 1, A T X N 1, A T X N 3, D M P K, F M R 1, F X N$, or HTT expansion (Supplementary

107 Table 1a); and 2) C9orf72 or FMR2 expansions of varying repeat lengths simulated using the

108 ART NGS read simulator ${ }^{26}$ (Supplementary Table 1b) as outlined in Supplementary Methods.

109 The simulated GS data were included in our analysis to assess the performance of the STR

110 algorithms on expansions of extremely high GC content (100\%) that may be refractory to

111 detection. 


\section{Patient Cohorts and ES and GS Data Generation}

113 The patient cohorts screened for known STR expansions in this study consist of the ES data of

114146 trios or quads from the Clinical Assessment of the Utility of Sequencing and Evaluation as a

115 Service (CAUSES) study and the GS data of 160 trios or quads from the Integrated

116 Metabolomics And Genomics In Neurodevelopment (IMAGINE) or CAUSES studies. Subjects

117 enrolled in the CAUSES study were children who were suspected on clinical grounds to have a

118 single gene disorder but in whom conventional testing had not identified a genetic cause.

119 Subjects enrolled in the IMAGINE study had impairment of motor function with onset before

120 birth or within the first year of life and additional clinical features that made perinatal

121 complications such as hypoxia or intracranial hemorrhage an unlikely explanation for their

122 problems. Most of the subjects enrolled in the CAUSES or IMAGINE studies had intellectual

123 disability. The ES or GS data from the unaffected parents were used to verify the inheritance or

124 unstable transmission of variants. These studies were approved by the Institutional Review

125 Board of the BC Children's and Women's Hospital and the University of British Columbia

126 (H15-00092 and H16-02126).

127 The trio/quad ES data were sequenced by Ambry Genetics (Aliso Viejo, United States),

128 Centogene (Rostock, Germany), or BC Cancer Agency Genome Sciences Centre (Vancouver,

129 Canada) to a mean coverage of $\sim 60 \mathrm{x}$. The library preparation protocols and sequencers used to

130 generate the trio/quad ES data are described in Supplementary Table 2.

The median coverage of the trio/quad GS data ranged from 36 to $80 \mathrm{x}$ and was generated

132 by the McGill University and Genome Quebec Innovation Centre (Quebec, Canada). GS libraries

133 were prepared using the NxSeq ${ }^{\circledR}$ AmpFREE Low DNA Library Kit Library Preparation Kit and 
134 Adaptors (Lucigen, Wisconsin, US) or xGen Dual Index UMI Adapters (Integrated DNA

135 Technologies, Coralville, US) and sequenced on an Illumina HiSeqX sequencer.

The paired-end reads (125 or $150 \mathrm{bp}$ ) of both the ES and GS datasets were aligned to the

137 UCSC hg19 human reference genome using BWA-MEM, and duplicates were marked with

138 Picard ${ }^{27}$. All patient ES data underwent single-nucleotide variant (SNV) and indel analysis, and

139145 out of the 146 trios or quads included in this study had no clinically-relevant SNV/indel

140 variants. We also analysed the ES data of a quad with known myotonic dystrophy (Type 1; DM1

141 - MIM 160900) in the proband and his mother as a positive control. Our patient GS data

142 underwent SNV, indel, structural, and mitochondrial variant analysis, with a causal variant

143 identified in about half of the trios (unpublished data). We included the GS data of all cases in

144 this study.

145 Bioinformatics Tools for STR Analysis

146 The STR analysis tools implemented in this study include lobSTR ${ }^{15}$, HipSTR ${ }^{28}$, RepeatSeq ${ }^{17}$,

147 TREDPARSE ${ }^{1}$, ExpansionHunter ${ }^{10 ; 19}$, GangSTR $^{18}$, STRetch $^{11}$, and exSTRa ${ }^{12}$. The key features

148 of these tools and the commands and parameters used to execute them are described in Table 1

149 and Supplementary Table 3, respectively. We first used ExpansionHunter (EH) version 2 in this

150 study $^{10}$ and later included the improved iteration (version 3) of EH optimized to genotype STRs

151 with complex or mixed repeat motifs ${ }^{19}$.

\section{Disease STR Catalogs}

153 The STR analysis tools assess known disease STRs included within a pre-defined catalog

154 supplied by the authors. The known pathogenic STR loci included in these catalogs, as well as

155 their allelic categories and corresponding repeat lengths, are summarized in Supplementary

156 Table 4. Notably, the region files for ExpansionHunter only included pre-defined OTS for FMRI 
and C9orf72 loci, while GangSTR included OTS in the region files of all 12 pathogenic STR loci

158 provided with the tool. Some of the region files of known disease STRs analysed in this study

$159(A R, A T N 1, F X N$, and FMR2) were missing for GangSTR. Therefore, we added these loci and

160 included their OTS as described in Mousavi et al. (2019) ${ }^{13}$.

\section{Interpretation of FMs and non-FMs}

162 The data from the genotyping methods were classified as "FM" if the estimated repeat lengths of

163 the STRs exceeded their respective FM thresholds (Supplementary Table 4). STRetch and

164 exSTRa calls were classified as "FM" if the $p$-values post-multiple-testing-adjustment were

165 significant $(<0.05)$. For STRetch, we used the control file (containing data from 143 healthy

166 individuals) provided with the tool.

\section{Decision Tree Classification}

168 Decision tree analysis is a supervised machine learning (ML) classification method ${ }^{29}$. We

169 employed this approach to infer the best model or the best combination of STR analysis tools to

170 detect FM expansions with optimal sensitivity and specificity. We used the Python Scikit-Learn

171 ML library ${ }^{30}$ to implement the decision tree classifier and used the STR calls from the

$172 \mathrm{EGA} /$ simulated GS to train and test the classifiers on the data from the Isaac and BWA

173 alignments.

174 For our preliminary decision tree analysis, we used the outputs generated using the

175 default parameters for each of the STR analysis tools. We compiled the results generated by the

176 STR analysis tools on the Isaac and BWA-aligned GS data. We labeled the EGA and simulated

177 genome's true STR expansion status or class label (FM or non-FM for a given locus).

178 Essentially, the single known or characterized STR expansion in each of the EGA and simulated

179 genomes was assigned to the "FM" class, while the status of the other STR loci was assigned to 
"non-FM". The data from the STR callers were then transformed into binary flags: 1 indicating at least one of the two alleles was called as "FM", and 0 indicating both alleles were "non-FM". From there, we removed all rows with missing values and supplied the data to the classifier. We divided our dataset into 80 and $20 \%$ to train and test the classifier, respectively, and then implemented the classifier. We used the Gini index approach to ascertain the efficiency of an attribute (i.e., the STR caller) in differentiating samples belonging to the FM and non-FM classes. To evaluate the performance of the classifier, we extracted different metrics, including precision (true positives $\mathrm{TP} /(\mathrm{TP}+$ false positives $[\mathrm{FP}]))$, recall $(\mathrm{TP} /(\mathrm{TP}+$ false negatives $[\mathrm{FN}]))$, accuracy, and F1-score $(2 *(($ precision*recall $) /($ precision+recall $)))$, and analysed the receiver operating characteristic (ROC) curve, a ratio of sensitivity $(\mathrm{TP} /(\mathrm{TP}+\mathrm{FN}))$ and inverted specificity $(1-(\mathrm{TN} /(\mathrm{TN}+\mathrm{FP})))$, and the precision-recall curve, a ratio of precision and recall or sensitivity. To avoid over-fitting of the data and to evaluate the robustness of the classifier, we performed 10-fold cross-validation on the training dataset and identified the best model for targeted disease STR analysis in both Isaac and BWA-aligned GS data.

195 performance of the STR analysis tools and the resultant decision tree model. First, we assessed the performance of ExpansionHunter with OTS on selected STR loci that are known to harbor

197 expansions exceeding sequencing fragment lengths. This was to retrieve unmapped and 198 mismapped IRR/IRR pairs and improve the repeat length estimation and detection of FMs.

199 Second, we used a PM or IM repeat length threshold instead of FM threshold for FMRI and 200 FMR2 STR loci to classify expanded alleles and documented the sensitivity as well as the FP 201 rates of the genotypers. Third, we tested exSTRa's performance on BWA-aligned GS with 202 control data from a cohort of 100 healthy individuals. We could not perform a similar analysis on 
203 Isaac-aligned GS due to the lack of Isaac-aligned GS data of healthy subjects. We carefully

204 evaluated how these parameter tweaks influenced the performance of the STR analysis tools and

205 selected the optimized outcomes to rerun our decision tree classifier. The precision, recall,

206 accuracy, and F1-score metrics of this newer model generated on the test dataset and cross-

207 validation on the training dataset were then compared to our preliminary decision tree analysis

208 with default parameters.

209 Screening for Known Disease STR Expansions in Patient Data

210 Finally, we screened our patient trio/quad ES and GS data for known disease STR expansions

211 using the tools identified by the classifier. Of the probands analysed in this study, 60 have had

212 clinical FMR1 STR testing, three have had clinical SCA STR panel tests, one has had a clinical

$213 F X N$ STR test, and four others have had clinical DMPK STR tests. All of these clinical PCR-

214 based STR tests were negative for a pathogenic expansion, except for a confirmed DMPK FM in

215 a proband and his mother. All individuals who were expansion-negative at the tested locus were

216 used as negative controls.

217 For all the expanded STRs identified in the patients, we analysed the parental genotype

218 calls to verify the inheritance or unstable transmission of the alleles. Subjects with potential

219 expansions of known disease STRs were identified for orthogonal validation to ascertain the

220 specificity of our decision tree. Molecular testing (PCR and capillary electrophoresis) of some of

221 the identified STR candidates was performed by Centogene (Germany). 


\section{RESULTS}

\section{Performance of STR Algorithms on Isaac versus BWA-aligned GS Data}

224 The lobSTR, HipSTR, RepeatSeq, EH versions 2 and 3, GangSTR, TREDPARSE, STRetch, and

225 exSTRa results of Isaac- and BWA-aligned EGA and simulated GS data are shown in

226 Supplementary Tables 5 and 6, respectively. The spanning-read-only algorithms (lobSTR,

227 HipSTR, and RepeatSeq) did not detect any FMs in either Isaac- or BWA-aligned GS data, as

228 expected. Therefore, we omitted these tools from all subsequent analyses.

The sensitivity of EH_v2 and EH_v3, GangSTR, TREDPARSE, STRetch, and exSTRa

run with default parameters in detecting FMs in Isaac- and BWA-aligned GS is summarized in

Table 2. EH_v2 and EH_v3, TREDPARSE, and STRetch exhibited consistent performance and

had a sensitivity of $\sim 70 \%$ in both Isaac and BWA alignments. GangSTR's sensitivity was better

233 on Isaac (55\%) compared to BWA (38\%) alignments. In marked contrast, exSTRa detected more

234 FMs in the BWA (88\%) than Isaac (56\%) alignments (see Supplementary Figures 1a and 1b for

235 exSTRa's plots on Isaac- and BWA-aligned GS, respectively). On Isaac-aligned data, STRetch,

236 EH_v2, and EH_v3 detected the most FMs, followed by TREDPARSE, exSTRa, and GangSTR.

237 On BWA-aligned data, exSTRa detected the most FMs, followed by STRetch, EH_v2, EH_v3,

238 TREDPARSE, and GangSTR. Notably, although exSTRa and STRetch detected more FMs, they

239 also had the most FP calls.

All FMs missed by the genotypers were under-sized and classified incorrectly as PM, IM,

241 or NL (Supplementary Tables 7a and 7b). Additional results on the performance of the

242 genotypers in classifying NL, IM, and PM alleles are included in Supplementary Tables 8 and 9

243 and Supplementary Results. Among the analysed STR loci, FMR1, FMR2, and homozygous

$244 F X N$ FMs were particularly refractory to detection (Supplementary Tables 7a and 7b). 


\section{Decision Tree Classification}

246 We first trained and tested the decision tree classifier on the generated default-parameter results

247 of EH_v2, EH_v3, GangSTR, TREDPARSE, STRetch, and exSTRa. After removing the rows

248 with missing values, the compiled STR calls of the Isaac- and BWA-aligned EGA and simulated

249 GS datasets had 1238 and 1232 rows (one row per sample per STR locus), respectively. In Isaac-

250 aligned data, EH_v2, which had the lowest Gini impurity or performed the best in classifying

251 alleles was assigned to the root node (node \#0) and correctly classified 47 out of 66 FMs and 918

252 out of 924 non-FMs in the training dataset (Supplementary Figure 2a). STRetch (node \#1) and

253 EH_v3 (node \#11) detected one of the FMs missed by EH_v2. In the test dataset, the decision

254 tree model had precision, recall, and F1-score of 100, 90, and 95\%, respectively, to detect FMs;

255 for non-FMs, the precision, recall, and F1-score were 99, 100, and 100\%, respectively. The ROC

256 and precision-recall plots are shown in Supplementary Figure 2b. The 10-fold cross-validation of

257 this model on the training dataset yielded a ROC_AUC (Area Under the Curve) of $85.48 \pm$

$258 \quad 12.58 \%$ (mean \pm standard deviation $)$

In the BWA-aligned data, EH_v3 at the root node correctly classified 43 out of 60 FMs

260 and 921 out of 925 non-FMs in the training dataset, with exSTRa and GangSTR recovering one

261 of the FMs missed by EH_v3 (Supplementary Figure 3a). The precision, recall, and F1-score to

262 detect FMs and non-FMs in the test data were 95, 81, and 88\% and 98, 100, and 99\%,

263 respectively. The ROC and precision-recall curves are shown in Supplementary Figure 3b. The

264 ROC_AUC metric of the model's 10-fold cross-validation on the training dataset was $86.24 \pm$

$2658.38 \%$.

266 In both Isaac and BWA analyses, nearly five out of the six features (STR tools)

267 contributed to the performance of the model (Supplementary Figures 2c and 3c), led by either 
EH_v2 or EH_v3. The sensitivity for detecting FMs in BWA-aligned data was slightly lower compared to the Isaac analysis. Overall, the decision tree classifier on the Isaac and BWA test datasets generated using the default-parameter settings missed 10 to $20 \%$ of the FMs. To improve the detection sensitivity, we evaluated some parameters that we believed might help

272 capture more of the true FMs.

273 Tested Parameters: First we tested the effect of including OTS in the detection of FMs. While 274 GangSTR's region files included OTS for all analysed loci, the author-supplied JSON files of 275 EH did not include OTS for $D M P K, F X N$, or FMR2 loci, which are known to harbor expansions 276 exceeding fragment lengths. In our initial EH run without OTS, we noted reduced sensitivity in 277 the detection of FXN and FMR2 FMs (Supplementary Table 7). Therefore, we added OTS for 278 analysing these loci with EH_v2, which helped identify two out of three FMR2 expansions in 279 both Isaac- and BWA-aligned data (Supplementary Table 10). For the FXN locus, there was no 280 improvement in sensitivity, highlighting the general limitation of the genotypers in reliably 281 detecting homozygous FXN FM expansions. Second, because the GC-rich expansions such as 282 those at the FMR1 locus tend to be under-sized owing to reduced coverage even in PCR-free 283 Illumina GS datasets ${ }^{10}$, we used an IM (54 repeats) and PM (60 repeats) repeat length threshold 284 for FMR1 and FMR2 loci, respectively, instead of their FM threshold (both at 200 repeats). With 285 this tweak, EH_v2 and EH_v3 detected all FMR1 and FMR2 FMs in Isaac- as well as BWA286 aligned data (Table 3). TREDPARSE detected 83 to $89 \%$ of the FMR1 FMs, but none of the 287 FMR2 FMs, while GangSTR detected 16 to $22 \%$ of the FMR1 FMs and none of the FMR2 FMs. 288 The identified FPs in this analysis include the known FMR1 PMs and a few borderline FMR1 IM 289 alleles that are closer to the threshold. Lastly, we hypothesized that adding data from a control 290 cohort to exSTRa's analysis of BWA alignments would further improve its FM detection 
potential. With controls, exSTRa yielded a sensitivity of $95 \%$ and detected all homozygous $F X N$

FM expansions, as well as all FMR1 and FMR2 FMs (Supplementary Figure 1c).

Of these parameters, using the IM/PM threshold for FMR1 and FMR2 genotype analysis

294 and performing exSTRa's BWA analysis with controls were useful in detecting refractory STR

training datasets, EH_v2 at the root node correctly classified all but one FM and most of the non-

297 FM alleles (Figures 1a and 2a). The classifier's precision, recall, and F1-score in the Isaac- and

BWA-aligned test datasets were 83,100 , and $91 \%$ and 90,100 , and $95 \%$ to detect FMs and 100,

98, and $99 \%$ and 100,99 , and $99 \%$ to detect non-FMs, respectively. The ROC and precision-

recall plots are shown in Figures $1 \mathrm{~b}$ and $2 \mathrm{~b}$. The ROC_AUC metric for cross-validation was

$95.14 \pm 5.12 \%$ for Isaac and $96.99 \pm 3.72 \%$ for BWA. All six STR analysis tools contributed to but GangSTR contributed to the performance of the classifier on the BWA-aligned GS (Figure

306 The decision rules that emerged from this analysis suggest the best approach to categorizing FMs

307 is to support EH_v2 and/or EH_v3 FM calls with (at least) one other tool (STRetch,

308 TREDPARSE, exSTRa, or GangSTR for Isaac, and STRetch or exSTRa for BWA).

309 Unsurprisingly, we also noticed a drop in precision due to the increase in FP counts, possibly

310 precipitated by the inaccurate identification of FMRI PM and some IM alleles. 


\section{Analysis of Known Disease STR Loci in Clinical NGS Data}

312 All our patient ES and GS data were BWA-aligned, so we followed the decision tree model

313 generated on the BWA-aligned EGA and simulated GS datasets, which suggested using EH_v2

314 and/or EH_v3 in addition to STRetch or exSTRa. We added some additional disease STR loci to

315 the EH_v2 variant catalog (Supplementary Table 4), analysing a total of 21 disease STRs using

316 all four tools in our patient cohort.

First, we identified 16 EH_v2 FM expansions that were supported by at least one of

EH_v3, STRetch, or exSTRa. Of the samples that were not called as expanded by EH_v2, we

319 screened for positive calls in EH_v3, STRetch, and exSTRa outputs. STRetch and exSTRa,

320 which had higher FP call rates in the EGA and simulated datasets, identified 298 and 442 disease

321 STR in our patient cohort. Therefore, any positive calls made on these two tools needed to be

322 supported by either EH_v2 or EH_v3. In total, we identified 27 samples, 17 with FM expansions

323 of the $A R, A T X N 1, A T X N 2, A T X N 8, D M P K, F X N, H T T$, or TBP locus, nine with IM or PM

324 alleles in the FMR1 locus, and one with a borderline allele in the ATXN2 locus (summarized in

325 Table 4). Supplementary Table 11 shows the EH_v2, EH_v3, STRetch, and exSTRa results of

326 the identified STR candidates.

We found that most probands with an identified STR candidate inherited the allele from a

328 parent, except for the ATXN1 FM in a proband (890-P) with 39 repeats (Supplementary Table

329 11) compared to the parental $A T X N 1$ NL alleles that had 28 to 31 repeats (data not shown). The

330 inherited expansions either remained unchanged or decreased by one or a few repeat units or

331 increased by 1 to $\sim 15$ repeats during intergenerational transmission. We also found seven FM

332 expansions in parents that were not inherited by the proband. 
All individuals who tested negative in their molecular assessments for $F M R 1, F X N, S C A$, or $D M P K$ FM expansions were also categorized as non-expanded by our bioinformatics workflow (data not shown). In the ES data of the proband (2010-P) and his mother (2010-M) with DM1 and a DMPK FM ( $>50$ repeats) finding on molecular assessment, EH_v2, EH_v3, and

337 exSTRa identified the FM expansion. However, the repeat length estimated by EH_v2 and

338 EH_v3 in 2010-P and 2010-M was 50 repeats, which is significantly lower than the molecular 339 findings of 150 repeats in 2010-P and 430 repeats in 2010-M (Supplementary Table 11). After 340 including OTS to EH's analysis of the DMPK locus, the FM estimate of EH_v2 and EH_v3 was $341 \sim 80$ repeats (data not shown).

Based on the repeat lengths estimated by EH_v2 and EH_v3, we categorized the

343 identified FMs as reduced- or full-penetrance (Table 4; the different repeat size ranges associated 344 with reduced- and full-penetrance of the STR expansion disorders are summarized in 345 Supplementary Table 4). Nine of the FMs we identified in the probands and parents were in the 346 fully-penetrant repeat size range, with another five in the reduced-penetrance range. The $A R$ FM 347 in a proband (1901-P) and her father (1901-F) was categorized as full-penetrance by EH_v3 (38 348 repeats) and reduced-penetrance by EH_v2 (37 repeats). We performed PCR-based molecular tests to verify the expansion status of a subset of the 350 identified FMs (molecular findings summarized in the last column of Table 4 and Supplementary 351 Table 11). The HTT FMs identified by EH_v2 (37 repeats), EH_v3 (37 repeats), STRetch, and 352 exSTRa in a proband (1530-P) and his father (1530-F) were concordant with the molecular test 353 (37 \pm 1 repeats). Also, the $A R$ FMs in a father (1901-F) and proband (1905-P) identified by 354 EH_v2 (37 repeats), EH_v3 (38 repeats), and STRetch were consistent with the PCR result (37 \pm 3551 repeats). On the other hand, the TBP FM in a mother (1992-M) identified by EH_v2 (52 
repeats) and EH_v3 (53 repeats) could not be verified by PCR (37 \pm 1 repeats). For the other

357 identified FMs with an unknown STR expansion status, we are currently performing molecular

358 validation.

359 Lastly, we investigated the genotype calls of the disease STRs made by EH_v2, EH_v3, 360 and GangSTR in our patient ES and GS datasets to see if the NL allele frequency distribution at

361 these loci agreed with the reported population frequencies of NL alleles (Supplementary Figures

3624 and 5, and Supplementary Table 12). In general, the repeat length distribution pattern of the

363 STR alleles for most loci was consistent across the ES (Supplementary Figure 4) and GS

364 (Supplementary Figure 5) data, except for the FMR1 and FMR2 loci, which were characterized

365 inconsistently in the ES data. EH_v3 genotyped fewer ATXN8 alleles and also had a different

366 repeat length distribution profile for the $A T X N 7$ and $H T T$ loci in the ES data. For the CSTB locus,

367 more 1-repeat genotype calls were made by the tools in the ES data, while we found none in the

368 GS data. More than half of the individuals in our clinical cohort are of European ancestry, so we

369 compared the frequency of the three most common alleles ascertained in the GS data to the

370 common NL allele in the Caucasian population reported in the literature (Supplementary Table

371 12). Except for a few loci, the repeat lengths of the most common alleles determined by the tools

372 were generally in good agreement with the reported repeat length of the common NL allele in the

373 Caucasian population.

\section{DISCUSSION}

375 The contribution of STR expansions to disease is just beginning to be understood. Hitherto, 40

376 neurological disorders have been found to have a causal STR expansion mutation underlying

377 their pathogenesis ${ }^{2}$, with some recent studies reporting the identification of novel pathogenic

378 STR expansions through NGS or the more advanced third-generation long-read sequencing 
379 technologies ${ }^{31-35}$. The challenges in detecting and characterizing the repeat lengths of STR

380 expansions in short-read NGS are well recognized ${ }^{36}$. However, recent algorithmic improvements

381 facilitate the detection of STR expansions that exceed read and/or fragment lengths, providing us

382 the opportunity to analyze a larger panel of known disease STR loci simultaneously through ES

383 and $\mathrm{GS}^{1 ; 10-14}$. Some of these methods may also be useful in scanning the entire genome or exome

384 for novel disease-causing STR expansions ${ }^{11 ; 13}$.

valuable for identifying disease-causing expansions because these programs leverage evidence

387 beyond the reads that span an STR, enabling the genotyping of larger repeat expansions. Other

388 methods like STRetch and exSTRa detect STR expansions but do not reliably genotype them

389 (STRetch) or do not genotype them at all (exSTRa). expansions mapped using two different aligners, Isaac and BWA, showed that the choice of aligner impacts the sensitivity of GangSTR and exSTRa. GangSTR performed better on Isaac alignments, whereas exSTRa performed better on BWA alignments. and the GC-rich FMR1 and FMR2 FMs were the most challenging. We modified some

396 parameters to increase the FM detection potential at these loci and found that exSTRa's

397 sensitivity improved with control datasets, detecting all FXN, FMR1, and FMR2 FMs in the 398 BWA-aligned data. Also, reducing the repeat length thresholds from FM to PM/IM size ranges 399 enabled the detection of FMR1 and/or FMR2 FMs with EH_v2, EH_v3, and TREDPARSE. 400 Using this reduced cut-off also might detect some IM and PM carriers who, although not 401 affected, may be at risk of having affected children if their IM/PM allele is highly unstable 
402 and/or susceptible to late-onset conditions ${ }^{37}$. Early detection and genetic counselling of these at-

403 risk individuals might, therefore, help IM/PM allele carriers make informed reproductive

404 decisions and avoid affected pregnancies ${ }^{37}$.

405 The ML decision tree analysis on the STR results generated using the afore-mentioned

406 parameter modifications detected all FMs with EH_v2 and/or EH_v3 with support from one

407 other tool (STRetch, TREDPARSE, exSTRa, or GangSTR for Isaac, and STRetch or exSTRa for

408 BWA). EH contributed significantly to the better overall performance of the classifier on both

409 Isaac and BWA alignments. Applying these decision rules to our clinical cohort, we identified 27

410 individuals with an expansion in a known disease STR locus. Of these, 17 individuals had an FM

411 expansion of the $A R, A T X N 1, A T X N 2, A T X N 8, D M P K, F X N, H T T$, or TBP locus, nine

412 individuals had an FMR I allele in the IM or PM size range, and one individual had a borderline

413 ATXN2 allele.

414 Using our approach, we were able to confirm the presence of a clinically-validated

$415 D M P K$ FM in the ES data of a proband and his mother with DM1 and also confirm the inherited

$416 H T T$ and AR FM in two families using clinical PCR and capillary electrophoresis. We classified

417 a TBP FM detected by EH_v2 and EH_v3, but unverified by PCR, as a false-positive.

418 Importantly, none of the 68 individuals who previously had a negative clinical $F M R 1, F X N$,

$419 S C A$, or HTT test result were falsely-identified as "expanded" by our computational workflow.

420 For the analysis of the DMPK locus with EH (the default catalog file of which does not

421 include OTS), we recommend including OTS as this could result in a significant improvement in

422 the repeat length estimation, particularly in the GS data, and yield clinically-relevant

423 information. Although the threshold for defining pathogenic DMPK FMs that cause DM1 is only

42450 repeats, the different clinical forms of DM1 (mild, classic, and congenital), associated with 
425 varying severity and age of onset of symptoms, are caused by DMPK FMs in the range of 50-

$426 \sim 150, \sim 100-\sim 1000$, and $>1000$ repeat units, respectively ${ }^{38}$. We show that with OTS, EH performs

427 better at sizing DMPK FMs that ranged from $~ 130$ to over 2000 repeats in the EGA GS data and

428 yields estimates that correlate better with the FM repeat lengths in these individuals

429 (Supplementary Figure 6).

430 Although the methods presented in this study perform well in detecting and sizing FMs,

431 for some disease STR loci, the difference between a non-FM and an FM, or between a reduced-

432 penetrance and full-penetrance FM is only a few repeat units, making it difficult to discriminate

433 these borderline alleles of clinical significance. This limitation is also inherent to PCR-based

434 tests as DNA polymerase slippage during STR amplification may result in under- or over-

435 estimation of an STR's size by one or two repeat units ${ }^{39}$.

436 In summary, implementation of a clinical bioinformatics workflow, such as the approach

437 outlined in this study, to screen for STR expansions in ES and GS data can help identify disease-

438 associated variants that would otherwise have gone undetected, promote cascade testing, and

439 improve diagnostics and treatment/management of repeat expansion disorders. 


\section{ACKNOWLEDGMENTS}

We would like to thank all the CAUSES and IMAGINE Study investigators. CAUSES

442 Study investigators include Shelin Adam, Christele Du Souich, Alison Elliott, Anna Lehman, Jill

443 Mwenifumbo, Tanya Nelson, Clara van Karnebeek, and Jan Friedman. The CAUSES Study is

444 funded by Mining for Miracles, British Columbia Children’s Hospital Foundation, and Genome

445 British Columbia. IMAGINE Study investigators include Patricia Birch, Madeline

446 Couse, Colleen Guimond, Anna Lehman, Jill Mwenifumbo, Clara van Karnebeek, and Jan

447 Friedman. The IMAGINE study is supported by the Canadian Institutes of Health

448 Research (CIHR - SCA-145104) through CHILD-BRIGHT (Child Health Initiative Limiting

449 Disability - Brain Research Improving Growth and Health Trajectories), with additional support

450 provided by BC Children's Hospital Foundation and the Michael Smith Foundation for Health

451 Research (MSFHR). Indhu Shree Rajan Babu is supported by the MSFHR Research Trainee

452 Award. We thank the Rare Disease Foundation for funding our research on developing a

453 bioinformatics pipeline to analyse STRs in next-generation sequencing data (Grant \# 2332). We

454 thank Compute Canada for the Research Allocation Competitions allocation, which facilitated

455 our analysis of the IMAGINE and EGA genomes, and Julia Handra for coordinating the STR

456 molecular testing of the clinical samples. 


\section{REFERENCES}

458 1. Tang, H., Kirkness, E.F., Lippert, C., Biggs, W.H., Fabani, M., Guzman, E., Ramakrishnan,

2. Sznajder, Ł., and Swanson, M.S. (2019). Short Tandem Repeat Expansions and RNAMediated Pathogenesis in Myotonic Dystrophy. Int J Mol Sci 20.

3. Paulson, H. (2018). Repeat expansion diseases. Handb Clin Neurol 147, 105-123.

4. Salcedo-Arellano, M.J., Dufour, B., McLennan, Y., Martinez-Cerdeno, V., and Hagerman, R. (2020). Fragile X syndrome and associated disorders: Clinical aspects and pathology. Neurobiol Dis 136, 104740.

5. Mila, M., Alvarez-Mora, M.I., Madrigal, I., and Rodriguez-Revenga, L. (2018). Fragile X syndrome: An overview and update of the FMR1 gene. Clin Genet 93, 197-205. of neurological disease. Neuron 77, 825-843. Opin Struct Biol 16, 351-358. 
[Internet]. Seattle (WA): University of Washington, Seattle; 1993-2019. Available from: https://www.ncbi.nlm.nih.gov/books/NBK535148/.

10. Dolzhenko, E., van Vugt, J.J.F.A., Shaw, R.J., Bekritsky, M.A., van Blitterswijk, M., Narzisi, G., Ajay, S.S., Rajan, V., Lajoie, B.R., Johnson, N.H., et al. (2017). Detection of long repeat expansions from PCR-free whole-genome sequence data. Genome Res 27, 18951903.

11. Dashnow, H., Lek, M., Phipson, B., Halman, A., Sadedin, S., Lonsdale, A., Davis, M., Lamont, P., Clayton, J.S., Laing, N.G., et al. (2018). STRetch: detecting and discovering pathogenic short tandem repeat expansions. Genome Biol 19, 121.

12. Tankard, R.M., Bennett, M.F., Degorski, P., Delatycki, M.B., Lockhart, P.J., and Bahlo, M. (2018). Detecting Expansions of Tandem Repeats in Cohorts Sequenced with Short-Read Sequencing Data. Am J Hum Genet 103, 858-873.

13. Mousavi, N., Shleizer-Burko, S., Yanicky, R., and Gymrek, M. (2019). Profiling the genomewide landscape of tandem repeat expansions. Nucleic Acids Res.

14. Dolzhenko, E., Deshpande, V., Schlesinger, F., Krusche, P., Petrovski, R., Chen, S., Emigsequence-graph based tool to analyze variation in short tandem repeat regions. Bioinformatics. profiler for personal genomes. Genome Res 22, 1154-1162.

16. Willems, T., Zielinski, D., Yuan, J., Gordon, A., Gymrek, M., and Erlich, Y. (2017). 592. 
17. Highnam, G., Franck, C., Martin, A., Stephens, C., Puthige, A., and Mittelman, D. (2013).

Accurate human microsatellite genotypes from high-throughput resequencing data using informed error profiles. Nucleic Acids Res 41, e32.

18. Mousavi, N., Shleizer-Burko, S., Yanicky, R., and Gymrek, M. (2019). Profiling the genomewide landscape of tandem repeat expansions. Nucleic Acids Res 47, e90.

19. Dolzhenko, E., Deshpande, V., Schlesinger, F., Krusche, P., Petrovski, R., Chen, S., Emigsequence-graph-based tool to analyze variation in short tandem repeat regions. Bioinformatics 35, 4754-4756.

20. Langmead, B., and Salzberg, S.L. (2012). Fast gapped-read alignment with Bowtie 2. Nat Methods 9, 357-359.

514 21. Gymrek, M., Willems, T., Reich, D., and Erlich, Y. (2017). Interpreting short tandem repeat variations in humans using mutational constraint. Nat Genet 49, 1495-1501.

22. Raczy, C., Petrovski, R., Saunders, C.T., Chorny, I., Kruglyak, S., Margulies, E.H., Chuang, H.Y., Källberg, M., Kumar, S.A., Liao, A., et al. (2013). Isaac: ultra-fast whole-genome

23. Li, H., and Durbin, R. (2009). Fast and accurate short read alignment with Burrows-Wheeler transform. Bioinformatics 25, 1754-1760.

24. https://gatk.broadinstitute.org/hc/en-us/articles/360035535912-Data-pre-processing-forvariant-discovery. 
26. Huang, W., Li, L., Myers, J.R., and Marth, G.T. (2012). ART: a next-generation sequencing read simulator. Bioinformatics 28, 593-594.

27. Picard Tools. Broad Institute. http://broadinstitute.github.io/picard/.

529 28. Willems, T., Gymrek, M., Highnam, G., Genomes Project, C., Mittelman, D., and Erlich, Y. (2014). The landscape of human STR variation. Genome Res 24, 1894-1904.

29. Krzywinski, M., and Altman, N. (2017). Classification and regression trees. Nature Methods $14,757-758$.

30. https://scikit-learn.org/stable/.

534 31. van Kuilenburg, A.B.P., Tarailo-Graovac, M., Richmond, P.A., Drögemöller, B.I., Pouladi,

32. Sone, J., Mitsuhashi, S., Fujita, A., Mizuguchi, T., Hamanaka, K., Mori, K., Koike, H., inclusion disease. Nat Genet 51, 1215-1221.

33. Tian, Y., Wang, J.L., Huang, W., Zeng, S., Jiao, B., Liu, Z., Chen, Z., Li, Y., Wang, Y., Min,

545 34. Florian, R.T., Kraft, F., Leitão, E., Kaya, S., Klebe, S., Magnin, E., van Rootselaar, A.F., 
35. Corbett, M.A., Kroes, T., Veneziano, L., Bennett, M.F., Florian, R., Schneider, A.L., repeat expansions in STARD7 in familial adult myoclonic epilepsy linked to chromosome 2. Nat Commun 10, 4920.

36. Mantere, T., Kersten, S., and Hoischen, A. (2019). Long-Read Sequencing Emerging in Medical Genetics. Front Genet 10, 426. [Updated 2019 Nov 21]. In: Adam MP, Ardinger HH, Pagon RA, et al., editors. Available from: https://www.ncbi.nlm.nih.gov/books/NBK1384/.

38. TD, Bird. Myotonic Dystrophy Type 1. 1999 Sep 17 [Updated 2019 Oct 3]. In: Adam MP, Ardinger HH, Pagon RA, et al., editors. GeneReviews ${ }^{\circledR}$ [Internet]. Seattle (WA): University of Washington, Seattle; 1993-2020. Available from: https:/www.ncbi.nlm.nih.gov/books/NBK1165/.

39. Raz, O., Biezuner, T., Spiro, A., Amir, S., Milo, L., Titelman, A., Onn, A., Chapal-Ilani, N., 
TABLE 1. Features of some publicly available STR analysis algorithms.

\begin{tabular}{|c|c|c|c|c|c|c|c|c|}
\hline Features & lobSTR & RepeatSeq & HipSTR & TREDPARSE & ExpansionHunter & STRetch & exSTRa & GangSTR \\
\hline $\begin{array}{l}\text { Outputs repeat } \\
\text { length? }\end{array}$ & Y & $\mathrm{Y}$ & $\mathrm{Y}$ & $\mathrm{Y}$ & $\mathrm{Y}$ & Y & & $\mathrm{Y}$ \\
\hline Sequencing reads & Single \& Paired-end & Single \& Paired-end & Single \& Paired-end & Paired-end & Paired-end & Paired-end & Paired-end & Paired-end \\
\hline $\begin{array}{c}\text { Sequencing } \\
\text { platforms supported }\end{array}$ & \begin{tabular}{|c|}
$\begin{array}{c}\text { Illumina, Sanger, } 454, \\
\text { and IonTorrent }\end{array}$ \\
\end{tabular} & Illumina & Illumina & Illumina & Illumina & Illumina & Illumina & Illumina \\
\hline $\begin{array}{c}\text { Library prep. } \\
\text { supported }\end{array}$ & PCR \& PCR-free & n.a. & PCR \& PCR-free & PCR \& PCR-free & PCR \& PCR-free & PCR \& PCR-free & PCR \& PCR-free & PCR \& PCR-free \\
\hline Library prep. (rcmd) & None & None & None & None & PCR-free & PCR-free & None & None \\
\hline Aligners (rcmd) & lobSTR, BWA-MEM & Novoalign, Bowtie 2 & Indel-sensitive aligner & None & None & None & Bowtie2 & None \\
\hline Analysis approach & Targeted \& GW & Targeted \& GW & Targeted \& GW & Targeted & Targeted & GW & Targeted \& GW & Targeted \& GW \\
\hline $\begin{array}{c}\begin{array}{c}\text { NGS data type } \\
\text { supported }\end{array} \\
\end{array}$ & GS & GS & GS & GS & GS & GS \& ES & GS \& ES & GS \& ES \\
\hline NGS data format & .bam or .fastq/fasta & bam & bam & bam & .bam or .cram & bam or fastq & bam & bam \\
\hline $\begin{array}{c}\text { Built-In stutter } \\
\text { correction model* }\end{array}$ & $\mathrm{Y}$ & & Y & $\mathrm{Y}$ & & & & \\
\hline Test of significance & & & & & & $\mathrm{Y}$ & $\mathrm{Y}$ & \\
\hline Read types used & Spanning & Spanning & Spanning & $\begin{array}{c}\begin{array}{c}\text { Spanning, flanking or } \\
\text { partial, paired-end } \\
\text { reads, IRR }\end{array} \\
\end{array}$ & \begin{tabular}{|} 
Spanning, flanking, \\
IRR/IRR pairs
\end{tabular} & Anchored IRR & $\begin{array}{c}\text { Flanking, anchored } \\
\text { IRR }\end{array}$ & $\begin{array}{l}\text { Spanning, flanking, } \\
\text { IRR/IRR pairs }\end{array}$ \\
\hline Phasing & & & $\mathrm{Y}$ & & & & & \\
\hline PL & $\mathrm{C}++$ & $\mathrm{C}++$ & $\mathrm{C}++$ & Python & $\mathrm{C}++$ & Java & Perl \& R & $\mathrm{C}++$ \\
\hline Sizing limitation & $\mathrm{RL}$ & $\mathrm{RL}$ & $\mathrm{RL}$ & FL & Not limited & FL & n.a. & Not limited \\
\hline Control dataset & Not required & Not required & Not required & Not required & Not required & Required & Not required & Not required \\
\hline Complex repeats & n.a. & n.a. & n.a. & n.a. & $\mathrm{Y}$ & n.a. & n.a. & $\mathrm{N}$ \\
\hline Output files & .vcf, .allelotype.stats & .repeatseq, .calls, .vcf &.$v c f$ &.$v c f$, json &.$v c f$, json,. $\log$ &. $\operatorname{tsv}$ & $\begin{array}{c}\text { p values, ECDF, tsum } \\
\text { plots }\end{array}$ &.$v c f$ \\
\hline $\begin{array}{c}\text { Customized regions } \\
\text { file }\end{array}$ & Possible & Possible & Possible & Possible & Possible & $\begin{array}{c}\text { Possible, but not } \\
\text { recommended. }\end{array}$ & Possible & Possible \\
\hline
\end{tabular}

*Corrects the noise (stutters) introduced during PCR amplification-based library preparation

Library prep: library preparation protocol; rcmd: recommended; PL: programming language used

Y: Feature included; N: Feature not included

n.a.: not applicable; GW: genome-wide; GS: genome sequencing; ES: exome sequencing; IRR: in-repeat reads; RL: read-length; FL: fragment-length; Not limited: not limited by either RL or FL; ECDF: Empirical Cumulative Distribution Function; t-sum: aggregated T statistic 
TABLE 2. Full-mutation (FM) samples detected in the Isaac- and BWA-aligned European Genome-phenome Archive (EGA) and simulated genomes by the STR tools (ExpansionHunter versions 2 and 3 (EH_v2 and EH_v3), GangSTR, TREDPARSE, STRetch, and exSTRa) implemented using default parameters. The analysed EGA and simulated dataset had 86 samples with at least one known FM allele. The number of true-positives detected by the tools, sensitivity, and the number of false-positives identified in our default analysis of the Isaac- and BWA-aligned genomes are shown.

\begin{tabular}{|c|c|c|c|c|c|c|c|c|}
\hline & \multicolumn{4}{|c|}{ Isaac } & \multicolumn{4}{|c|}{ BWA } \\
\hline & $\begin{array}{c}\text { Detected FM } \\
\text { Samples }\end{array}$ & $\begin{array}{l}\text { True FM } \\
\text { Samples }\end{array}$ & Sensitivity & False-Positives & $\begin{array}{c}\text { Detected FM } \\
\text { Samples }\end{array}$ & $\begin{array}{l}\text { True FM } \\
\text { Samples }\end{array}$ & Sensitivity & False-Positives \\
\hline EH_v2 & 65 & 86 & 0.755813953 & 6 & 64 & 86 & 0.744186047 & 6 \\
\hline EH_v3 & 64 & 86 & 0.744186047 & 5 & 64 & 86 & 0.744186047 & 5 \\
\hline GangSTR & 47 & 86 & 0.546511628 & 8 & 33 & 86 & 0.38372093 & 8 \\
\hline TREDPARSE & 62 & 86 & 0.720930233 & 3 & 62 & 86 & 0.720930233 & 10 \\
\hline STRetch & 65 & 86 & 0.755813953 & 26 & 65 & 86 & 0.755813953 & 26 \\
\hline exSTRa & 48 & 86 & 0.558139535 & 33 & 76 & 86 & 0.88372093 & 35 \\
\hline
\end{tabular}


TABLE 3. Classification of the FMR1 and FMR2 ExpansionHunter versions 2 and 3 (EH_v2 and EH_v3), GangSTR, and TREDPARSE genotype calls using lowered thresholds to detect FMs in the Isaac- and BWA-aligned EGA and simulated genomes of samples with known FMR1 and FMR2 FM expansions. The number of FMs misclassified as normal (NL) or intermediate (IM) alleles are shown. The true number (n) of known FM alleles in the FMR1 and FMR2 genes is indicated in parenthesis. False-positive (FP) calls made by the tools are also reported.

\begin{tabular}{|c|c|c|c|c|c|c|c|c|c|c|c|c|c|c|}
\hline & \multicolumn{7}{|c|}{ Isaac } & \multicolumn{7}{|c|}{ BWA } \\
\hline & \multicolumn{4}{|c|}{ FMR1 $(\mathrm{n}=18)$} & \multicolumn{3}{|c|}{ FMR2 $(\mathrm{n}=3)$} & \multicolumn{4}{|c|}{ FMR1 $(\mathrm{n}=18)$} & \multicolumn{3}{|c|}{$F M R 2(\mathrm{n}=3)$} \\
\hline FM Threshold & \multicolumn{4}{|c|}{54 repeats } & \multicolumn{3}{|c|}{60 repeats } & \multicolumn{4}{|c|}{54 repeats } & \multicolumn{3}{|c|}{60 repeats } \\
\hline Allelic classification & FM & IM & NL & FP & FM & NL & FP & FM & IM & NL & FP & FM & NL & FP \\
\hline EH_v2 & 18 &. & & 20 & 3 & & 2 & 18 &. & 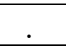 & 16 & 3 & . & 0 \\
\hline EH_v3 & 18 & . & 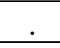 & 22 & 3 & . & 0 & 18 & . & . & 22 & 3 &. & 0 \\
\hline GangSTR & 4 & . & 14 & 7 & 0 & 3 & 0 & 3 & . & 15 & 0 & 0 & 3 & 0 \\
\hline TREDPARSE & 15 & 1 & 2 & 8 & 0 & 3 & 0 & 16 & 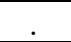 & 2 & 13 & 0 & 3 & 0 \\
\hline
\end{tabular}


TABLE 4. STR candidates identified in our patient cohort. Probands with an identified STR candidate are given a "-P" suffix in the "Sample ID" column, siblings, "-S", mother, "-M", and father, "-F". The genes harboring the STR candidate identified by our bioinformatics workflow and the inheritance pattern deciphered by comparing the proband's STR call with that of the parents are reported. "Sequencing" column shows the technology used: genome sequencing (GS) or exome sequencing (ES). The "Pathogenic SNV/indel/SV Finding" column indicates whether the proband has had a definite, probable, certain, or no diagnosis of a single nucleotide variant (SNV), indel, or structural variant (SV). Phenotypic presentations reported in the probands, STR Finding from our bioinformatics analysis, and the results from the molecular validation (if available) are also presented.

\begin{tabular}{|c|c|c|c|c|c|c|c|}
\hline Sample ID & Gene & Inheritance & Sequencing & $\begin{array}{l}\text { Pathogenic } \\
\text { SNV/indel/SV Finding }\end{array}$ & Phenotype detail & STR Finding & $\begin{array}{l}\text { Molecular } \\
\text { Validation }\end{array}$ \\
\hline 1901-P & $A R$ & Inherited & GS & No & $\begin{array}{l}\text { Short stature, delayed gross motor, speech and language development, spasiticity, cerebral } \\
\text { palsy, and hypertonia }\end{array}$ & \multirow[t]{2}{*}{$\mathrm{FM}(\mathrm{RP} / \mathrm{FP})$} & FM (RP) \\
\hline 1901-F & $A R$ & & GS & & & & FM (RP) \\
\hline 890-P & ATXN1 & De novo & ES & No & $\begin{array}{l}\text { Optic atrophy, findings suggestive of congenital stationary night blindness, growth restriction, } \\
\text { no dysmorphic features, and diffuse mild hypomyelination }\end{array}$ & $\mathrm{FM}(\mathrm{FP})$ & Pending \\
\hline 532-M & ATXN1 & & GS & & & FM (FP) & Pending \\
\hline $2560-\mathrm{M}$ & $A T X N 1$ & & ES & & & FM (FP) & Pending \\
\hline $1411-\mathrm{F}$ & ATXN1 & & ES & & & FM (FP) & Pending \\
\hline 821-P & $A T X N 2$ & Inherited & ES & No & $\begin{array}{l}\text { Mild intellectual disabilities, systemic hypertension, cutis aplasia, congenital heart defect, limb } \\
\text { anomalies, significant family history of her father with alopecia, learning problems, early onset } \\
\text { hypertension, and differential diagnosis of autosomal dominant Adams-Oliver syndrome }\end{array}$ & FM (FP) & Pending \\
\hline $821-\mathrm{M}$ & ATXN2 & . & ES & & & borderline ${ }^{\wedge}$ & Pending \\
\hline 1099-P & ATXN8 & * & ES & No & $\begin{array}{l}\text { Hearing loss, cataract, myopia, visceral (kidney and spleen) cysts, proteinuria, and dysmorphic } \\
\text { facial features }\end{array}$ & FM (RP) & Pending \\
\hline 235-P & ATXN8 & Inherited & GS & No & $\begin{array}{l}\text { Mild to moderate intellectual disability, history of psychosis, family history: a sister who also } \\
\text { has intellectual disability and history of psychosis, and a brother with mild developmental } \\
\text { delays }\end{array}$ & FM (RP) & Pending \\
\hline $235-\mathrm{M}$ & ATXN8 & & GS & & & FM (RP) & Pending \\
\hline 2010-P & $D M P K$ & Inherited & ES & Definite & $\begin{array}{l}\text { Myotonic dystrophy type } 1 \text {, inguinal hernias, joint hypermobility, strabismus, mild intellectual } \\
\text { disability, and dysmorphic facial features }\end{array}$ & $\mathrm{FM}(\mathrm{FP})$ & $\mathrm{FM}(\mathrm{FP})$ \\
\hline $2010-\mathrm{M}$ & $D M P K$ & & ES & & & FM (FP) & FM (FP) \\
\hline 699-M & FMRI & & GS & & & PM & Pending \\
\hline 148-M & FMRI & & GS & & & PM & $\begin{array}{l}\text { Pending (Proband } \\
\text { is negative for } \\
\text { FMR1 FM) }\end{array}$ \\
\hline $800-\mathrm{F}$ & FMRI & & GS & & & IM or PM & Pending \\
\hline 800-P & FMRI & Inherited & GS & Definite & $\begin{array}{l}\text { Macrocephaly, seizures, optic nerve hypoplasia, hyporeflexia, profound intellectual disability, } \\
\text { cortical visual impairment, and spastic tetraplegia }\end{array}$ & IM or PM & Pending \\
\hline $480-\mathrm{P}$ & FMRI & Inherited & GS & Probable & $\begin{array}{l}\text { Moderate intellectual disability, language delay, autism, borderline macrocephaly, low set ears, } \\
\text { down slanting palpebral fissures, high palate, and soft skin }\end{array}$ & IM or PM & Pending \\
\hline 712-M & FMRI & & GS & & & IM or PM & $\begin{array}{l}\text { Pending (Proband } \\
\text { is negative for } \\
\text { FMRI FM) }\end{array}$ \\
\hline 925-P & FMRI & Inherited & GS & No & $\begin{array}{l}\text { Intellectual disability, developmental delay including speech delay, dysmorphic features, and } \\
\text { behavioural challenges }\end{array}$ & NL or PM & Negative for FM \\
\hline $925-\mathrm{S}$ & FMRI & Inherited & GS & No & Intellectual disability, autism, developmental delay, and dysmorphic features & IM & Pending \\
\hline $925-\mathrm{M}$ & FMR1 & & GS & & & PM & Pending \\
\hline $1987-\mathrm{F}$ & FXN & & GS & & & $\mathrm{NL} / \mathrm{FM}$ & Pending \\
\hline 1530-P & HTT & Inherited & GS & Uncertain & $\begin{array}{l}\text { Global developmental delay, seizures, gliosis, developmental regression, encephalomalacia, } \\
\text { hirsutism, nystagmus, optic atrophy, cyanosis, abnormal muscle tone, scoliosis, hearing } \\
\text { impairment, and otitis media }\end{array}$ & FM (RP) & $\mathrm{FM}(\mathrm{RP})$ \\
\hline $1530-\mathrm{F}$ & HTT & & GS & & & FM (RP) & $\mathrm{FM}(\mathrm{RP})$ \\
\hline 1992-M & $T B P$ & & GS & & & FM (FP) & Negative for FM \\
\hline $2990-\mathrm{M}$ & $T B P$ & & ES & & & FM (FP) & Pending \\
\hline
\end{tabular}

RP: reduced penetrance; FP: full penetrance

*Father was not tested

${ }^{\wedge} \mathrm{RP}$ alleles have 33-34 repeats and FP alleles have $>=37$ repeats 
Figure 1. Decision tree classification of the STR calls of the Isaac-aligned EGA and simulated genome sequence (GS) data by ExpansionHunter versions 2 and 3 (EH_v2 and EH_v3), GangSTR, TREDPARSE, STRetch, and exSTRa using modified parameters. Panel (A) shows the decision tree generated by the classifier on the training dataset. Node \#0 at the top of the tree is the root node. Each node lists an STR tool (feature). The "samples" number represents the total number of data points present within a particular node, and "value" shows the number of expanded (or full-mutation or FM) and non-expanded (non-FM) data points. The shade of the colour of each node reflects the proportion of expanded to non-expanded data points, with deeper blue and orange meaning more nonexpanded and expanded data points, respectively. Gini index shows the impurity at each node. The terminal nodes shown in the last rows are the leaves. Leaves with a Gini of 0 have data points belonging to either the expanded or the non-expanded class. Panel B shows the ROC and precision-recall plots generated by the classifier on the test dataset. Panel C shows the ranking of the STR tools that contributed to the decision tree model. 
A
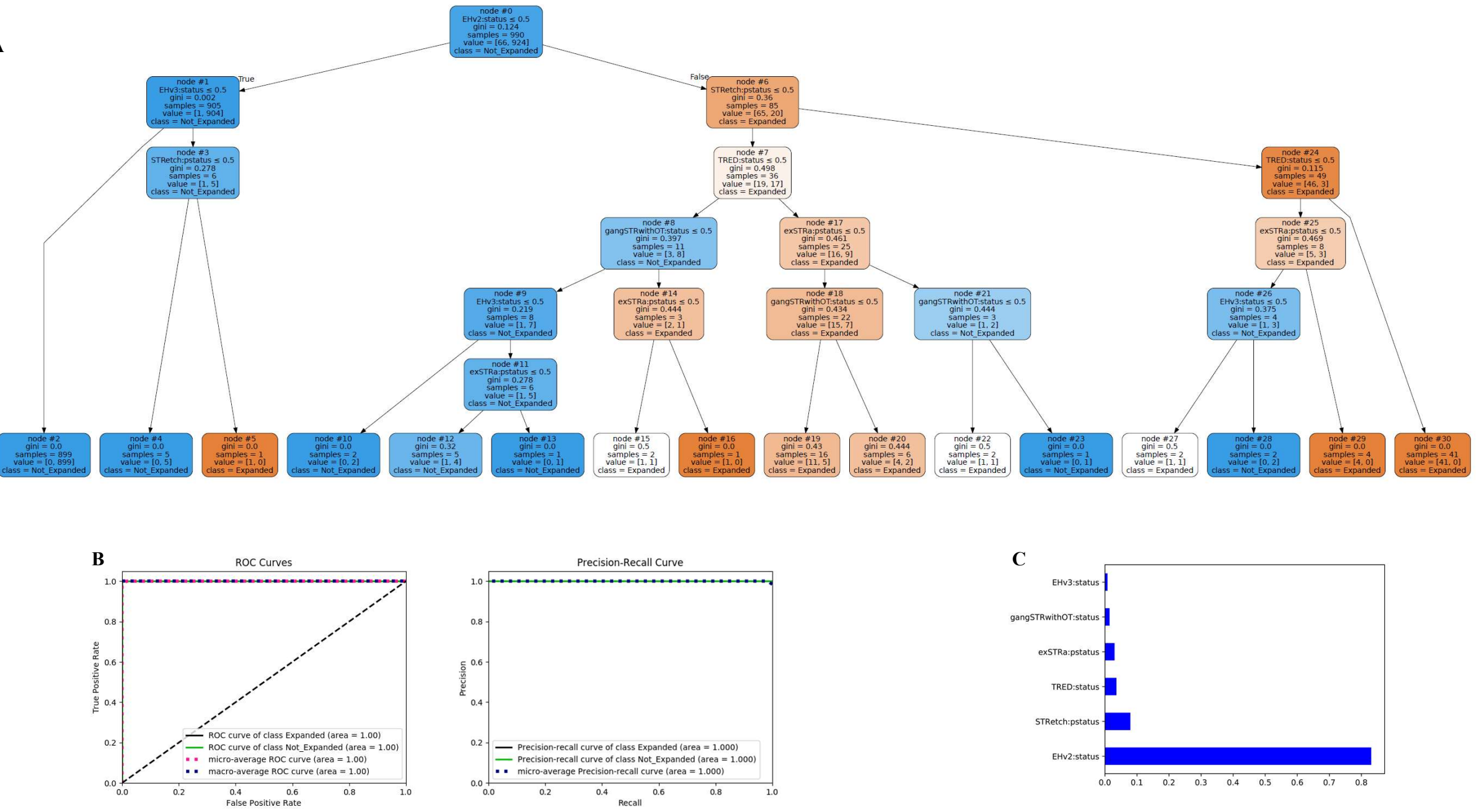
Figure 2. Decision tree classification of the STR calls of the BWA-aligned EGA and simulated GS data by ExpansionHunter versions 2 and 3 (EH v2 and EH v3), GangSTR, TREDPARSE, STRetch, and exSTRa using modified parameters. The decision tree generated by the classifier on the training dataset (A), ROC and precision-recall plots generated by the classifier on the test dataset (B) and ranking of the STR tools that contributed to the decision tree model (C) are shown.
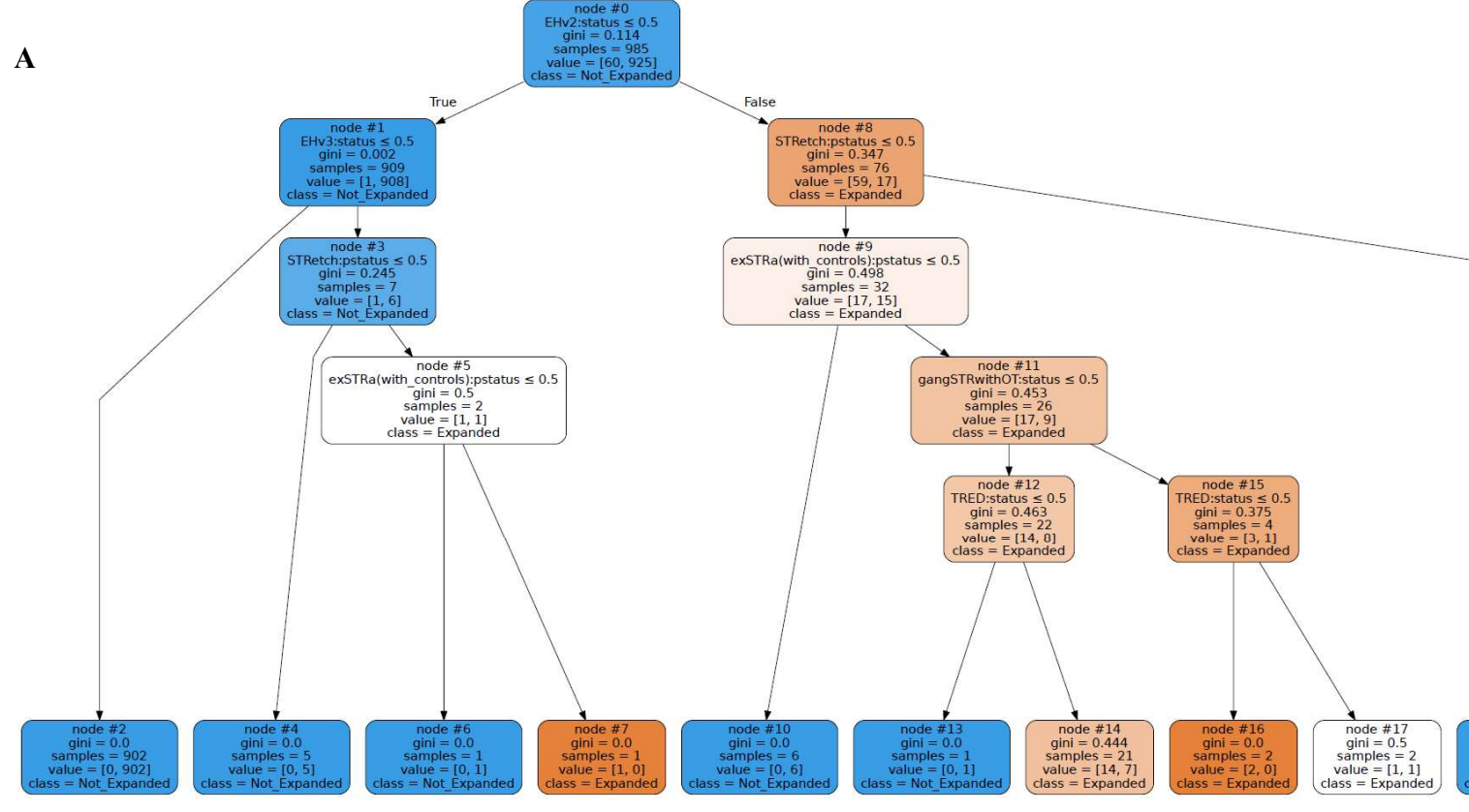

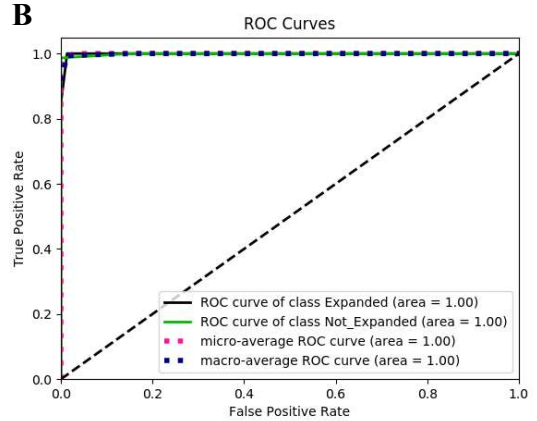

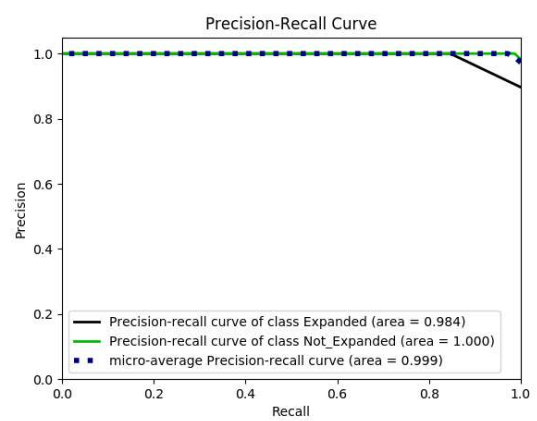
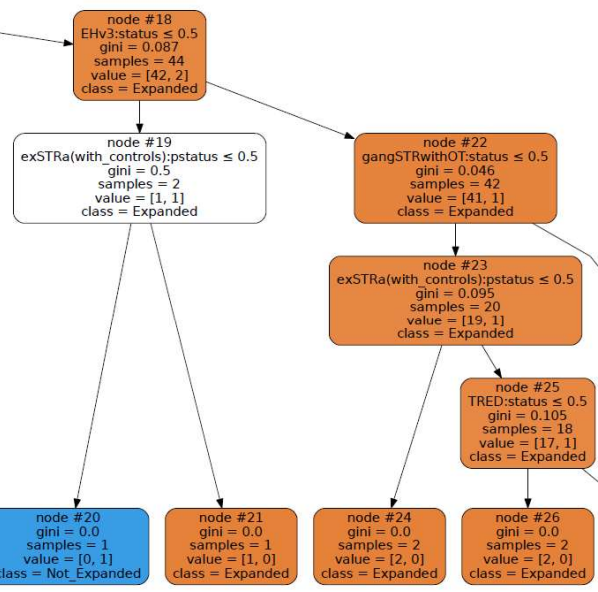

C

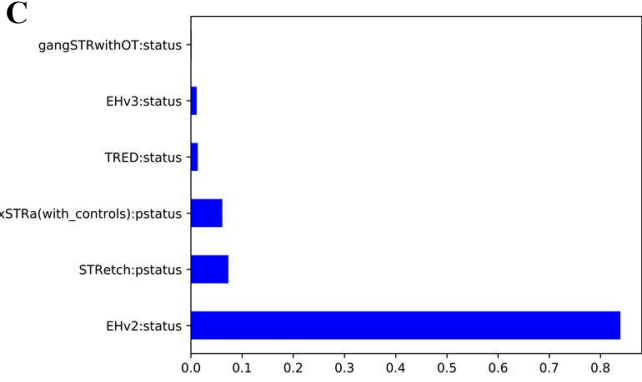

